\title{
The Mental Health Development in Malaysia: History, Current Issue and Future Development
}

\author{
Sheau Tsuey Chong ${ }^{1}$, M. S. Mohamad ${ }^{1} \&$ A. C. Er $^{2}$ \\ ${ }^{1}$ School of Psychology and Human Development, Faculty of Social Sciences and Humanities, Universiti \\ Kebangsaan Malaysia, Malaysia \\ 2 School of Social, Development and Environmental Studies, Faculty of Social Sciences and Humanities, \\ Universiti Kebangsaan Malaysia, Malaysia \\ Correspondence: Sheau Tsuey Chong, School of Psychology and Human Development, Faculty of Social \\ Sciences and Humanities, Universiti Kebangsaan Malaysia, 43600 UKM Bangi, Selangor, Malaysia. Tel: \\ 60-3-8921-4847. E-mail: stchong@ukm.my
}

Received: March 14, 2013 Accepted: April 11, 2013 Online Published: April 28, 2013

doi:10.5539/ass.v9n6p1 URL: http://dx.doi.org/10.5539/ass.v9n6p1

\begin{abstract}
In recent years, the burden of caregiving for mental illness service users has been alarming due to the lack of resources in mental health services in Malaysia. The issue of caregiving for mental illness service users in Malaysia now then become more complex because of its multiracial society, with people from different cultural backgrounds and who possess varied beliefs and attitudes. This paper aims to review the development of mental health services from the era of independence till the post millennium era. Firstly, the researchers discuss the history of mental health development by looking at mental health institution from the perspective of government plan. Secondly, the development of mental health policy is followed. Thirdly, mental health resources in terms of professional service providers and physical support are reviewed in this section. Lastly, current issues on mental health service and recommendation for future research. In conclusion, we suggest that it will be more effective for mental health professionals to join together to take collective action through the existing organisations such as the Malaysian Social Workers' Association, Malaysian Mental Health Association and Malaysian Psychiatric Association. Furthermore, mental health professionals can help the caregivers organise their own family support groups through partnership in order to voice their opinions to the government/policy makers for the improvement and development of social policy.
\end{abstract}

Keywords: mental health, history, Malaysian development, community health, caregivers

\section{Overview of Mental Health Development in Malaysia}

Malaysia sits at the heart of South East Asia with a population of 23 million people of diverse ethnicity, cultures and religious backgrounds. In 2000, it was reported that about 10.7 percent of the population had been diagnosed with mental illness (Jamaiyah, 2000). These numbers are quite high and only based on hospital figures. Therefore, the actual number of people living with mental illness nationwide remains unknown. However, the findings of some other studies carried out in both urban and rural areas in Malaysia, have also placed the prevalence of mental illness in the population between 9.6 per cent and 35 percent, respectively (Crabtree \& Chong, 2000). Is this the exact prevalence rate of mental illness in Malaysia? Of course, much can be debated about the reported and the actual figures. What appears to be eminently clear, however, is that there is a lack of resources in mental health services in Malaysia.

\section{The History of Mental Health Services in Malaysia}

Malaysian mental health service is a relatively young industry yet the number of mental health patients has grown rapidly for the past decades. Research in many countries has found that the act of taking care for any family member with serious mental illness living at home is stressful. The experience of caregiving may vary according to whether they are living in urban or rural areas and with cultural beliefs about mental illness especially in developing countries like Malaysia. There is a significant improvement of the provisions for mental health services in Malaysia today since independence from colonial rule in 1957. Here we discuss the improvement along with the history of Malaysia in threesubsections, i.e. rural vs city, East vs West Malaysia, 
general health (focusing on mental health services).

Initially, city health care was largely based on a system created to provide health servicesto plantation workers, whose health was of utmost importance for industries such as rubber, oil palm plantations and tin mines. During that time, rural or remote areas that had no connection with these industries were excluded from health care provision. Immediately after independence, the health care systems in Malaysia improved remarkably to embark provisions and institutions of medical care services. Only when the First Malaysia Plan (1966-1970) was introduced, rural people started to get benefits of health care where new health care facilities were established and scores of new medical professionals were trained. Another priority of the First Malaysia Plan was the improvement of public health services that were made available to citizens across the country. These efforts vastly improved the imbalance between health care in the cities and that which was available in the countryside (Ministry of Health, 1997). The Second Malaysia Plan (1970 - 1980) was set up to promote the welfare of all citizens, and particularly to improve the living conditions in rural areas.

Looking at the development of East and West Malaysia, medical facilities in East Malaysia (Sabah and Sarawak) were less well equipped and understaffed than those in West Malaysia (Peninsular)due to the lack of basic infrastructure and medical personnel (Crabtree \& Chong, 2000).In addition, the Ministry of Health, Malaysia $(\mathrm{MOH})$ began building more facilities in rural locations including midwife centres, dental clinics, mobile clinics and district hospitals to increase the health of the population. Moreover, the first Malaysia National Development Plans also introduced the Rural Health Service (RHS) in an attempt to increase access to medical facilities in rural areas and upgraded district hospitals facilities, to enable them to handle referrals from clinics all over the country. By the early 1980 s, in terms of the quality of healthcare the differences between the rural and city areas had been narrowed immensely albeit not eliminated entirely.

Now we discuss the development of mental health services in Malaysia. The set up of the first 'lunatic asylum' in small scale at the Penang Hospital in the north of Peninsular Malaysia in the late 1890s witnessed the start of mental health service. Here, sailors of the colonial navy with mental illness were treated. In 1910, there were records of a psychiatric hospital in the Taiping Hospital, which located in north of Peninsular Malaysia. Later, the Federal Lunatic Asylum near TanjongRambutan, Perak was established in 1911 with 280 beds. It was renamed Central Mental Hospital (CMH) in 1928 (Haque, 2005). In 1935, the Permai Hospital in Johor was built to cater for the needs of the people in the south of the country. In Sabah and Sarawak, two such hospitals were established in the 1920s. Although these facilities were designed as a hospital, treatment remained custodial in nature and the mental health service users continued to increase with a high incidence of mortality.

The revolution of mental health care provision in the country started when the first mental health ward was opened at the Penang General Hospital in 1958. That was the first time mental health service users were treated in a non-psychiatric hospital (Haque, 2000). In 1967, the first community-based rehabilitation of mentally ill persons was opened in Ipoh, Perak as a day-care centre. The first psychiatrist in Malaysia, a graduate of the UK, started practising in 1961. In 1975, three locally trained psychiatrists graduated from the University of Malaya (UM), and by 1995, there were almost 100 psychiatrists servicing in Malaysia (Crabtree \& Chong, 2000). As the nationwide development of health services was placed under the Ministry of Health $(\mathrm{MOH})$, it began to embark on a plan to decentralise health services, particularly the mental health services. Psychiatric care, which was strictly institutionalised in four mental hospitals (Bahagia Hospital, Permai Hospital, Sentosa Hospital and Bukit Padang Hospital), was confined to between 500 to 5,000 service users.Now, all other government hospitals are provided with one psychiatric unit to handle local mental health service users before being transferred to these institutions (Merican et al., 2004).Today,Malaysiahas 128 government hospitals with the availability of beds ranging from 4,770 to over 30,000 bedsand six mental health institutions (Ministry of Health, 2007).There are also a total of six non-governmenthospitals with 2,886 bedsand 233 private hospitals andnursing homes with11,637 beds. With a wider network of health care systems, mental health care is now morereadilyavailable, a marked comparison from the four mental hospitals decades ago. There are now 28 general hospitals with 22 equipped with community-based mental health servicesandover 100 outpatient primary care centres throughout the country (Ministry of Health, 2009a).

\section{Concepts of Mental Health in Malaysia}

The World Health Organisation (WHO) (2001: 5) defines mental health as "a state of well-being in which the individual realises his or her own abilities, can cope with the normal stresses of life, can work productively and fruitfully, and is able to make a contribution to his or her community". However, in Malaysia,the Ministry of Health (1997: 2) has defined mental health as "the capacity of the individual, the group and environment to interact with one another to promote subjective well-being and optimal functioning, and the use of cognitive, 
affective and relational abilities, towards the achievement of individual and collective goals consistent with justice". We can see this definition is different from WHO. The definition by Ministry of Health shows more collective nature. This all traces back to the context of multi-cultural country of Malaysia, with each culture thriving on caring and giving, providing trust and social support, and in many ways, always generous towards those who are needy and disabled (Deva, 2004).

Malaysia comprises three main ethnic groups: the Malays (66.1\%), Chinese $(25.3 \%)$ and Indians $(7.4 \%)$. The Malays are the indigenous people of Malaysia and follow the teachings of Islam. The majority of the Chinese claim affiliation with Christianity and Buddhism, while a sizeable number of Indians are Hindus. Therefore, the concepts of mental illness and mental health in Malaysia can and have been addressed from different perspectives, representing the influence of various races and religious beliefs.

The cultural assumption has brought a different definition of "mental health" that may have a different perspective on what mental health really is. The concept of mental health amongst Malays can be summarised as heredity, periodicity, congenital, brain strain, stress (including interpersonal), susceptibility, infection, contagion, delayed onset, conditioning, and resistance (Resner \& Hartog, 1970). The parallelism between folk and modern concepts suggests certain universal bases and clues in treating the mental health service users in the Malay culture (Resner \& Hartog, 1970).

The concept of mental health amongst Indians is centralised on the concept of mind-body dichotomy (Wig, 1999). To understand this concept, perhaps more important than any one quote is the broad Hindu view of life as summed up in the four aims of life - "Dharma", "Kama", "Artha" and "Moksha" (Wig, 1999). Dharma is righteousness, virtue or religious duty. Kama refers to the fulfilment of individual biological needs or sensual pleasures. Artha is the fulfilment of individual social needs and includes material gain, acquisition of wealth and social recognition. Moksha refers to liberation or release from worldly bondage and union with the ultimate reality. These aims provide harmony of life in which dharma appears to be the central axis. Indians who are Hindu believe that a person living with mental illness is the long-term result of imbalance between these four elements (Wig, 1999).

The Chinese concepts of mental health are influenced by the traditional Chinese medical beliefs based on Confucianism and Taoism (Yip, 2005a). Confucianism refers to a Chinese ethical and philosophical system of moral, social, political, philosophical, and quasi-religious thought that has had tremendous influence on the culture and history of East Asia (Yip, 2005a). Taoism refers to numerous teachings in the Tao TeChing and Zhuangziphilosophies and has never been a unified religion (Yip, 2005a). There are four principles of Taoism "Tao", "De (te)", "Wu wei" and "Pu". Tao literally means 'the way' to the natural, eternal, spontaneous and indescribable. It is rarely an object of worship, being treated more like the Indian concepts of atman and dharma. $D e$ (te) is "power; virtue; integrity", that is, the active living, or cultivation, of Tao. Wu wei is a central concept of Taoism and literally means 'without action'. It is in alignment with Tao, revealing the soft and invisible power within all things where the universe works harmoniously according to its own ways. The harmony is disrupted when someone exerts their will against the world. $P u$ is a symbol for a state of pure potential and perception without prejudice. It is usually seen as keeping oneself in the primordial state of Tao. It is believed to be the true nature of the mind, unburdened by knowledge or experiences. These principles refer to the concept of Yin and Yang as the symbol of life. Yip (2005a) noted that the Chinese believe that a person with mental illness is because of the imbalance between Yin-Yang as a result of the interaction of the Taoism principles.

In the Malay culture, spiritual and religious factors are viewed as vital parts in mental health. Generally, the labelling of mental illness as "sakitjiwa" or literally translated as illness of the soul among the Malays is common. Some of the Malay families believe that mental illness is not merely regarded as a medical illness but as a spirit possession or as a social punishment (Deva, 2004). It might be because of the Malay cultural belief systems that have strong connotations with supernatural causes of mental illness such as charms and evil deeds by jealous people, transgressions on religious-spiritual realms by the sufferer and also the weakness of the spirit of a person. These cultural beliefs lead the people to seek guidance from traditional healers called "bomoh" or "pawang" who are considered possessors of hidden knowledge and who can rid the individual of the possession and cure mental illness. The strong influence of culture in the Malay society results in the general concept that mental illness is an outcome of abandoning or neglecting traditional values (Haque\&Masuan, 2002).

In the Chinese culture, illness (both mental and physical) is believed to be caused by the lack of spirit or the weakness of yin and yang. They consider the emotions in a person as the integral aspects of the body's basic functions, which are regulated by the circulation of $c h$ ' $i$ (air or breath), that is partly innate and partly a product of one's food and drink. Ch' $i$ not only maintains the physical body but also the mental and spiritual processes in 
the individual. Abnormal emotions affect the functions of the $c h ' i$, for example, anger makes the $c h ' i$ rise; joy relaxes it; sorrow dissipates it; fear makes it go down; cold contracts it; heat makes it leak out; fright makes its motion chaotic; exhaustion consumes it; and worry congeals it (Haque, 2005). All these values play important roles in Chinese society. Traditionalism among them is strongly rooted, whereby primary allegiance is to the parents or to the family into which one is born. The Chinese believe that mental illness is caused by problems related to self-worthiness, which is measured by the material achievement (includes education, occupation and monetary gain) that brings the expected honour to the family (Haque, 2005;Bentelspacher et al., 1994).

In Indian culture, the strong influence of Hinduism regarding illness and treatment can be found in the Hindu textbook called the Vedas (Book of Knowledge). Indians believe that maladies resulting from supernatural causes can be treated using ayurveda (Pflug, 1992). Ayurveda is a complex philosophy that sees life as consisting of 24 elements that comprise three entities, sattva (mind); atma (soul); and sharira (body). The concept of mental illness among the Indians is a combination of physical and mental factors that needs balance from these three entities. In addition, Indians believe that evildoers could cast a spell on an individual to make them ill (Haque, 2005).

Therefore, the concepts of mental illness and mental health continue to be based on mythology, and are socially or culturally unacceptable in Malaysia. Mental health professionals in Malaysia have to consider the cultural sensitivity of the different faiths and belief systems, and accept that these people are deeply influenced by their culture, religious and traditional backgrounds.

\section{Mental Health Policy}

Malaysia's mental health policy was based on the British and Indian mental health laws in the early 1900s (Haque, 2005). Both West and East Malaysia introduced their own laws to govern mental health. These included the Mental Health Disorders Ordinance of 1952, which covered the Peninsular; the Lunatic Ordinance of Sabah of 1953; and the Mental Health Ordinance of Sarawak of 1961. In 1993, the Care Centres Act was introduced as a supplement to all previous acts on mental health. These acts addressed mental health issues separately and were only relevant to a certain part of Malaysia. It is clear that these acts were not comprehensive and remained outdated for a long period (Deva, 2004).

In 2001, the MOH introduced the new National Mental Health Act, which can be considered as a great achievement for the mental health system, as it meets the first part of the first recommendation by the WHO (1975). However, there were some concerns raised against the Act including that the act treats mental health as a medical problem rather than a phenomenon that is triggered by a whole range of psychosocial factors within the larger society (Crabtree \& Chong, 2000). The Mental Health Act 2001 encompasses detailed policy guidelines for the delivery of mental health services in Malaysia. This act has brought huge changes to the health professionals, the service users, and all those who are directly or indirectly affected by the mental health system in Malaysia.

In addition, the $\mathrm{MOH}$ also developed the National Mental Health Policy in 1997. This mental health policy provides comprehensive strategies and guidelines to address issues in mental health. All programmes and activities that are related to mental health are subsumed under the promotion of mental health, prevention of mental disorders, treatment of people with mental disorders and psychosocial rehabilitation. Some mental health issues such as accessibility and equity in services; comprehensiveness; continuity and integration; multi-sector collaboration; community participation; human resources and training; standards and monitoring; research; and legislation reviews have been covered in this new policy. This policy has three aims: (1) to provide a basis in developing strategies and direction to those involved in any planning and implementation towards improving mental health and well being; (2) to improve mental health services for populations at risk of developing psychosocial problems; and (3) to improve the psychiatric services for people with a mental disorder in the provision of care and protection by the family, community and relevant agencies. In other words, this policy is to promote community mental health care in Malaysia.

In 2003, the MOH introduced the National Mental Health Registry (NHMR), specifically for the monitoring of the population in Malaysia diagnosed with schizophrenia (Aziz et al., 2008). The NHMR collects information about patients with a mental disorder in Malaysia, which enables the MOH to estimate the incidence of selected mental disorders, and to evaluate risk factors and treatments available in the country. The first NHMR report, known as the NHMR Report 2003-2004, focused on schizophrenia and provides detailed information concerning the profile of persons with schizophrenia who registered with the psychiatry and mental health providers throughout Malaysia. The National Mental Health Registry has five objectives, namely, (1) to determine the disease burden attributable to mental disorders by quantifying its morbidity, and its geographic and temporal 
trends in Malaysia; (2) to identify subgroups in the population at high risk of mental disorders to whom prevention effort should be targeted; (3) to identify potential risk factors involved in mental disorders; (4) to evaluate the treatment, control and prevention of mental disorders; and (5) to stimulate and facilitate epidemiological research on mental disorders. All these mental health policies were developed by the MOH to oversee the needs of the practitioners, mental health service users and their families in providing the necessary facilities required by each group.

\section{Current Issues and Future Development}

Community mental health care has become an accepted form of treatment of a person with severe mental illness in the National Mental Health Act, 2001. Malaysia has also moved towards treating psychiatry service users in community-based care, especially in their own house supported by their family. In 2000, the Ministry of Health launched the psychosocial treatment programmes in community clinics. A year later, the National Mental Health Act was amended to include a new strategy promoting community mental health care services, which resulted in many mental health service users being discharged into the community with support available at the community clinics and general hospitals. Caring for and supporting people with mental illness in the least restrictive environment, and preferably with their family, is now the focus of the Malaysian National Mental Health Strategy. The current mental health policy supports the continuing process of deinstitutionalisation, that is, the movement from institutional to community care along with the integration of psychiatric care into the mainstream general health care system (Ministry of Health, 1997).

Generally, community mental health services can be divided into two categories, formal and informal (WHO, 2001). Formal community mental health services usually link with primary care services and informal care provided in a community. These services include community-based rehabilitation services, hospital diversion programmes, mobile crisis teams, therapeutic and residential supervised services, and home help and support services in the community. Community mental health services are not based in a hospital setting but work closely with mental and general hospitals, which are really in need of staff with a high level of skills and training in mental health. These services provide the opportunity for many persons with mental illness to continue living in the community, thus promoting community integrated care. Another category is informal community mental health services. This service may be provided by local community members other than general health professionals including traditional healers, community support groups and NGOs. Informal providers are a useful complement for formal mental health services, which can be important in improving the outcomes of persons with mental illness. Such service providers usually have high acceptability and accessibility in the community.

In Malaysia, the $\mathrm{MOH}$ has started psychosocial programmes in community clinics to provide the opportunity for people who have mental illness and their relatives to achieve a quality of life through their own environment. The government believes that the empowerment of mentally ill persons and their families can be obtained through the new process of training and skills development (Merican et al., 2004). It is important to make sure that people with mental illness can live efficiently in the community. This process really needs the involvement of mental health service users, families, community members and health professionals (Merican et al., 2004). Unfortunately, the MOH faced some difficulties in its attempt to create comprehensive mental health care because of the scarcity of funds (Ministry of Health, 2007). As a result of this budgetary restriction, it is clear that comprehensive community care is unlikely to be a viable option without the support of primary and secondary care services. Therefore, to overcome this problem, the MOH has used the integration of mental health care into general health services, specifically at the primary health care level (Jamaiyah, 2000). These are aimed to improve the treatment of the mental and physical problems of those diagnosed with schizophrenia. For example, less stigmatisation of mental health service users, as mental and behavioural disorders are being seen and managed alongside physical health problems in community clinics or general hospitals. It may also provide various advantages to the $\mathrm{MOH}$, including a shared infrastructure leading to cost-efficiency savings, the potential to provide universal coverage of mental health care, and importantly, the use of community resources, which can partly offset the limited availability of mental health personnel.

Internationally, studies have shown that family caregivers face considerable difficulty due to the lack of coping resources within the community, and that they appear to be unprepared and untrained as caregivers for their relatives with severe mental illness (Grad \& Sainsbury, 1963; Biegel et al., 1991; Lefley, 1996; Jenkins \& Schumacher, 1999; Wong et al., 2004; Chang \&Horrocks, 2006; Liu et al., 2007). Numerous researchers in Western countries reported that historically, many families living with mental illness service users were not well treated by the society or by the health professionals (Freeman \& Simmons, 1963; Biegel et al., 1991; Lefley, 1996; Doornbos, 1997; Addington et al., 2005). Quite a lot of studies in the Western world demonstrated that 
family members report and complain of the burden and distress, anxiety, depression and economic strain while playing a major role in the care of their relatives (Schulze \&Rossler, 2005; Hall, 2000; Saunders, 2003; Barrowclough et al., 1996; Schene et al., 1994; Szmukler, 1996).

In Eastern culture, families have been implicitly or explicitly blamed for causing their relative's illness (Lee et al., 2005). Some of the researchers noted that family caregivers in East and Southeast Asia, who perceived a higher level of caregiver burdens, were those who lived in a family with poorer functioning, worse health status and less satisfaction with their social support (Chang \&Horrocks, 2006; Chien et al., 2005; Lee et. al, 2005; Lee \& Sung, 1998).

In Malaysia, since the Malaysian government discharged long-stay mental health service users into the community, family caregivers have been regarded as being responsible for looking after their relatives. Traditionally, Malaysian families choose to look after their ill members at home and see the hospital as a last choice (Deva, 2004). However, the situation is quite different with mental illness. Deva (2004) noted that families of severely mentally ill relatives prefer their relatives to be in the institution rather than at home. One possible reason is a misunderstanding or lack of knowledge about mental health and illness, and devotion to the traditional cultural belief systems among multi-ethnic/religious groups (Malaysian Psychiatry Association, 2005). This may pose challenges to the mental health service users and their families as well as service providers. As discussed earlier, the meaning of mental illness is often interpreted by Malaysians as possession by evil spirits, a moral weakness or punishment from a higher being or God (Haque, 2005; Deva, 2004; Resner\&Hartog, 1970). In addition, those relatives who are diagnosed with schizophrenia are commonly perceived as restless, violent and unpredictable even when they are only occasionally symptomatic (Merican et al., 2004). Some Malaysians stereotyped mental health service users as dangerous, violent and beyond professional help (Malaysian Psychiatric Association, 2005). This situation poses a big challenge to Malaysian family caregivers who take on the role of caregivers to a person with severe mental illness (Chang \&Horrocks, 2006).

\section{Conclusion}

Since the Malaysian government promoted community mental health care, many families are taking care of their relatives in their community. However, the lack of knowledge and lower mental health literacy and devotion to the traditional multicultural belief systems pose challenges to family caregivers, mental health service users and service providers. The mental health issue is more alarming because of the lack of community mental health facilities, especially in the rural areas, whilst the number of family caregivers looking after their relatives is increasing. There are plenty of studies about family caregiving of mental illness in Western cultures but they are still lacking in the Eastern cultures. Hence, these studies have not provided an understanding of the experiences of multi-racial family caregivers, especially in developing countries such as Malaysia. The multidimensional experience of caregiving remains unexplored in Malaysia. Therefore, more studies are much needed, as highlighted by the service providers and caregivers themselves when the researcher was involved in mental health social work practice.

\section{Acknowledgments}

The authors would like to thank UniversitiKebangsaan Malaysia for UKM-DLP-2012-030 under the leadership of the Assoc. Prof. Dr. Er Ah Choy.

\section{References}

Addington, J., McCleery, A., \& Addington, D. (2005). Three-year outcome of family work in an early psychosis program. Schizophrenia Research, 79(1), 107-116. http://dx.doi.org/10.1016/j.schres.2005.03.019

Aziz, A. A., Salina, A. A., Abdul, K. A. B., Badiah, Y., Cheah, Y. C., Nor, H. A., .. Chee, K. Y. (2008). The national mental health registry (NMHR). TheMedical Journal of Malaysia, 63, 15-17.

Bentelspacher, C. E., Chitran, S., \& Rahman, M. A. (1994). Coping and adaptation patterns about Chinese, Indian and Malay families caring for a mentally ill relative. The Journal of Contemporary Human Service, 75, 287-294.

Biegel, D. E., Sales, E., \& Schulz, R. (1991). Family Caregiving in Chronic Illness: Alzheimer's disease, Cancer, Heart Disease, Mental Illness, and Stroke. Family caregiver applications series, 1. Newbury Park, Calif: Sage Publications.

Chang, K. H., \& Horrocks, S. (2006). Lived experiences of family caregivers of mentally ill relatives. Journal of Advanced Nursing, 53(4), 435-443. http://dx.doi.org/10.1111/j.1365-2648.2006.03732.x 
Chien, W. T., Chan. S., Morrissey, J., \& Thompson, D. (2005). Effectiveness of a mutual support group for families of patients with schizophrenia. Journal of Advanced Nursing, 51(6), 595-608. http://dx.doi.org/10.1111/j.1365-2648.2005.03545.x

Crabtree, S., \& Chong, G. (2000). Standing at the crossroads: Mental health in Malaysia since independence. In A. Haque (Ed.), Mental Health in Malaysia: Issues and Concerns. Kuala Lumpur: University Malaysia Press.

Deva, P. M. (2004). Malaysia mental health country profile. International Review of Psychiatry, 16, $167-176$. http://dx.doi.org/10.1080/09540260310001635203

Doornbos, M. (1997). The problems and coping methods of caregivers of young adults with mental illness. Journal of Psychosocial Nursing and Mental Health Services, 35(9), 22-26.

Freeman, H., \& Simmons, O. (1963). The mental patient comes home. New York: John Wiley.

Grad, J., \& Sainsbury, P. (1963). Mental illness and the family. Lancet, 1, 544-547. http://dx.doi.org/10.1016/S0140-6736(63)91339-4

Haque, A. (2004). Religion and mental health: The case of American Muslims. Journal of Religion and Health, 43(1), 45-58. http://dx.doi.org/10.1023/B:JORH.0000009755.25256.71

Haque, A. (2005). Mental health concepts and program development in Malaysia. Journal of Mental Health, 14(2), 183-195. http://dx.doi.org/10.1080/09638230500059997

Haque, A., \& Masuan, K. A. (2002). Religious psychology in Malaysia. International Journal for the Psychology of Religion, 12, 277-289. http://dx.doi.org/10.1207/S15327582IJPR1204_05

Jamaiyah, H. (2000). Community mental health in Malaysia: Marriage of psychiatry and public health. Buletin Kesihatan Masyarakat Isu Khas, 155-166.

Jenkins, J. H., \& Schumacher, J. G. (1999). Family burden of schizophrenia and depressive illness. The British Journal of Psychiatry, 174, 31-38. http://dx.doi.org/10.1192/bjp.174.1.31

Lee, S., Lee, M. T. Y., Chiu, M. Y. L., \& Kleinman, A. (2005). Experience of social stigma by people with schizophrenia in Hong Kong. The British Journal of Psychiatry, 186, 153-157. http://dx.doi.org/10.1192/bjp.186.2.153

Lee, Y., \& Sung, K. (1998). Cultural influences on caregiving burden: Cases of Koreans and Americans. The International Journal of Aging \& Human Development, 46(2), 125. http://dx.doi.org/10.2190/PM2C-V93R-NE8H-JWGV

Lefley, H. P. (1996). Family Caregiving in Mental Illness. Family Caregiver Applications Series, 7. Newbury Park, Calif: Sage Publications.

Liu, M., Lambert, C. E., \& Lambert, V. A. (2007). Caregiver burden and coping patterns of Chinese parents of a child with a mental illness. International Journal of Mental Health Nursing, 16(2), 86-95. http://dx.doi.org/10.1111/j.1447-0349.2007.00451.x

Malaysian Psychiatric Association. (2005). Survey: Stigma keeps millions with anxiety disorders from treatment. Retrieved April 12, 2010, from http://www.psychiatry-malaysia.org/html/consensus_intro.shtml

Merican, M. I., Rohaizat, Y., \& Haniza, S. (2004). Developing the Malaysian health system to meet the challenges of the future. Medical Journal of Malaysia, 59(1).

Ministry of Health. (1997). National Health and Morbidity Survey. Kuala Lumpur: Public Health Institute.

Ministry of Health. (2007). Health Facts 2006. Ministry of Health Malaysia. Retrieved March 2, 2008, from http:/www.moh.gov.my/images/gallery/stats/heal_fact/health_facts_2006.pdf

Ministry of Health. (2009a). Clinical Practice Guidelines: Management of Schizophrenia in Adults. Ministry of Health Malaysia. Retrieved August 12, 2010, from http://www.moh.gov.my/v/mhe

Pflug, B. (1992). Education in ayurveda: A re-constructional analysis. New Delhi: Gian Publishing House.

Resner, G., \& Hartog, J. (1970). Concept and terminology of mental disorder among Malays. Journal of Cross-cultural Psychology, 1(4), 369-382. http://dx.doi.org/10.1177/135910457000100409

Saunders, J. C. (2003). Families living with severe mental illness. Issues in Mental Health Nursing, 24(2), 175-198. http://dx.doi.org/10.1080/01612840305301 
Schene, A. H., Tessler, R. C., \& Gamache, G. M. (1994). Instruments measuring family or caregiver burden in severe mental illness. Social Psychiatry and Psychiatric Epidemiology, 29(5), 228-240. http://dx.doi.org/10.1007/BF00796381

Schulze, B., \& Rossler, W. (2005). Caregiver burden in mental illness: Review of measurement, findings and interventions in 2004-2005. Current Opinion in Psychiatry, 18(6), 684-691. http://dx.doi.org/10.1097/01.yco.0000179504.87613.00

Szmukler, G. (1996). From family 'burden' to caregiving. Psychiatric Bulletin, 20(8), 449. http://dx.doi.org/10.1192/pb.20.8.449

Wig, N. N. (1999). Mental health and spiritual values. A view from the East. International Review of Psychiatry, 11(2-3), 92-96. http://dx.doi.org/10.1080/09540269974230

Wong, D., Tsui, H., Pearson, V., Chen, E., \& Chiu, S. (2004). Family burdens, Chinese health beliefs, and the mental health of Chinese caregivers in Hong Kong. Transcultural Psychiatry, 41(4), 497-513. http://dx.doi.org/10.1177/1363461504047932

World Health Organization. (2001). Mental Health: New Understanding, New Hope. Geneva: World Health Organization.

Yip, K. S. (2005). Family caregiving of clients with mental illness in the People's Republic of China. International Journal of Psychosocial Rehabilitation, 10(1), 35-42. 